$\xi=-1$

\title{
ICR: information, cluster and route agent based method for efficient routing in VANET
}

\author{
V. Gokula Krishnan ${ }^{1}$ *, Dr. N. Sankar Ram ${ }^{2}$ \\ ${ }^{1}$ PhD Research Scholar Faculty of Computer Science \& Engineering Sathyabama University \\ ${ }^{2}$ Professor Department of Computer Science Engineering Sri Ram Engineering College \\ *Corresponding authorE-mail: gokul_kris143@yahoo.com
}

\begin{abstract}
Vehicular Ad-Hoc Network (VANET) is a variety of mobile ad-hoc networks (MANET) that differs from the fact that VANETS communicates between vehicle-to-vehicle and vehicle-to-roadside communication network. VANETs have the possibility to integrate with $\mathrm{WiFi}$, Bluetooth and other connectivity protocols which makes it a new standard. The desired outcome expected from VANET is to communicate between nodes in any environment irrespective of traffic density and vehicle locations. Since vehicular communication is a part of constantly changing environment and it must also operate in urban and rural areas. Taking in mind the large number of nodes that are actually participating in these networks and also the property of high mobility between them, it has resulted in many debates about a feasible routing protocol. This paper introduces a multi-agent system approach to solve the problems faced by vehicular communications since it comprises of more than one agent solve the problem of routing, the resulting protocol gives higher efficiency and better performance on packet delivery and end-to-end packet delay.
\end{abstract}

\section{Introduction}

When it comes to mass transportation, all over the world, publiclyowned vehicles will remain dominant irrespective of its drawbacks like accidents, congestion as well as negative environmental impact. Leveraging the information as well as communication technologies can improve road safety, reduce delays, enhance the traveler experience, etc. Creating a Mobile Ad hoc Network (MANET) is one such possibility where vehicles themselves act as network nodes. VANET [1] is another such technology in which the temporary mobile nodes are associated and also manage themselves independently without any fixed infrastructure. Even if there are some connections to the nodes, they will not affect the decisions of the network management.

On the other hand, due to the unpredictable environment of vehicles, all the traditional routing protocols which are applied for MANETs cannot be applied to VANETs[3][4]. Hence, during the recent years, many new routing protocols have been proposed explicitly for VANETs which deals with its unique characteristics and also aim at routing the information within vehicular as well as road-side nodes. The primary target of routing in VANETs is to transmit data in a unicast manner, i.e., from a single source to a single destination, or in geocast manner, i.e., by amalgamating wireless multi-hop and carry-forward techniques. But measuring the effectiveness and efficiency is always challenging in case of these protocols.

There are mainly 3 categories for the architecture of VANET namely vehicle to vehicle ad hoc networks, vehicle to infrastructure (e,g., fixed gateways at the road sides ) and hybrid architecture.

VANETs finds its application in many areas and its scope is huge. Firstly, Co-operative Message Transfer [2], which allows the slow/stopped vehicles to exchange messages with other vehicles for co-operation and help. Reliability and latency is a major concern in this case and it may automate emergency braking for avoiding accidents. Next is Post Crash Notification, in which a vehicle involved in an accident will broadcast warning messages containing its locations to the trailing vehicles to take a decision within a short time and to highway patrol for tow away support. Road Hazard Control Notification is another application where cars notify other cars about any hazard such as landslides, road feature because of road curve or sudden downhill. Another application is Traffic Vigilance in which cameras can be installed in the Road Side Unit (RSU) to input and act as a tool for low or zero tolerance campaign for driving offenses. Remote Vehicle Personalization/Diagnostics help in downloading personalized settings for a vehicle or in uploading vehicle diagnostics from/to other infrastructures. VANETs can act as a medium which gives Internet Access through RSU in case if the RSU can work as a router. Digital Map Downloading is also an application of VANET where map regions can be downloaded by drivers based on requirement prior to their travel to a new area, and also Content Map Database Download can get valuable information from mobile hot spots or home stations acting as a portal. On-demand movie experience can be expanded to the vehicles by the application of VANETs in Real Time Video Relay. Service providers can profit from Value-added advertisement by attracting them to their stores. Announcemnts such as petrol pumps, highway restaurants can also reach the vehicles, even in the absence of internet. Route Diversions can be updated to oncoming vehicles in case of congestion. Lastly, even in case of Toll Collection, it can be paid electronically using VANETs. In case of Multi-agent systems [17], various aspects can be considered for the benefit of the vehicles such as cooperation, distribution, intelligence as well as autonomy. As the architecture grows in a distributed and dynamic environment, this can get more interesting. Therefore, a routing protocol for the VANET can 
solve a lot of problems in case of the before mentioned application.

\section{Related work}

Major challenge faced by Vehicular Communication is routing. Researchers have proposed many different types of routing protocols [5] [6]. In VANET, the routing protocol can be classified into many methods based on the related work that was carried out Road based routing and Communication based routing are two types. Road based routing methods are available to developed areas life urban, highways as well as rural area too. In case of urban scenario, bidirectional communication and high density have added features. The high density of vehicles results in a reduction in the mobility of vehicles during communication, but Highway routing takes up unidirectional communication as well as low vehicle density. Rural routing method is less efficient due to the low network density and high mobility.

Additionally, few more routing protocols [7] [8] are Position based routing, Topology based routing, and Cluster based routing with regards to the arrangement of the nodes. Position based routing tracks the location information of the vehicles using Global Positioning System (GPS) technology. It however does not maintain a routing table, but instead uses the location information of the source node, next-hop node and destination node to carry out the routing. Topology based routing can be further subcategorised into Proactive, Reactive and Hybrid routing. Proactive routing transfers data from source node to destination node using the shortest path algorithm. It is a table driven approach as the information in the table changes periodically when there is a change in mobility. Therefore, procative is highly appropriate for low mobility and fewer topology change areas in VANET. Reactive on the other hands used ondemand methods to deliver data. The routing table in this case is reconfigured frequently. This can be adapted to the environment which has high mobility and dynamic changes in topology. Hybrid routing is a combination of the Proactive and Reactive routing. It delivers efficiency and scalability during the process of communication. In Cluster based routing [10] [12] groups of vehicles combine together to form clusters and is managed by a Cluster Head (CH). $\mathrm{CH}$ takes responsibility to coordinate the vehicles in inter-cluster and intra-cluster structures and also sends the packets to the next hop node during the process of routing. Various approaches are proposed to elect the $\mathrm{CH}$. This method is highly scalable.The protocols proposed for MANET cluster cannot be adapted for VANET due to high mobility in the nodes. Hence some routing protocol [14] such as CBR, COIN and LORA_CBF are proposed in order to adapt to the VANET environment.

Based on the modes of communication between the nodes the routing can be further classified as Geocast, Broadcast and Multicast routing. Geocast routing coordinates the position based and multicast based routing methods. This method sends packets to all vehicles in a specific location or area using Zone of Relevance (ZOR). This reduces the overhead in network and ensures reliability in delivery of packets. The broadcast routing on the other hand sends the packets to all the nodes as and when the destination is out of the range for communication. Intelligent Transport System (ITS) uses mostly the Broadcast and Geocast routing to share details such as climate, traffic and emergency warning signal across to all the vehicles. This method enables to get reliable transmission of packet and thereby minimizing the network overhead. But sending the packets to every node in the network leads to replication of those packets. Similarly, another scenario can be inferred from Multicast routing, in which,the source node sends the packet to multiple destination. The routing of these packets can be carried out in tree based and mesh based routing structure. But the problem arises when there are frequent changes in the network. These changes apparently result in change of tree structure and also the mesh structure, thereby affects the performance in a negative way. The mobility of nodes [19] in VANETs require the backbone of the network to reconstruct continuously in a periodic fashion. Thus there arises a critical research on more distributed, adaptive and intelligent protocol. Agent technology [16] has found its way into the most exciting research area in case of VANETs. Kumar et al proposed an algorithm in VANET for agent learning-based clustering. They have evaluated the performance of this algorithm by taking various metrics such as node participation, connectivity percentage, period of cluster head, connectivity preservation ratio as well as a message transmission ratio. Tyagi et al [18] on the other hand proposed an architecture exclusively for a VANET using mobile agent to design clusters. Mobile agents [5] among VANET nodes are used to create a concept of clustering. One of the vehicle acting as cluster head takes all the decisions for other vehicles. Since there is always a change in the topology, the decisions taken might not be valid for a longer time. All these collections of drawbacks act as a driving force to develop a efficient routing method which will improve the packet delivery ratio and thereby reduces end to end delay in vehicular communication.

\section{Proposed system}

The proposed system comprises about a multi-agent routing protocol, which includes three agents, namely Information Agent (IA), Cluster Agent (CA) and Route Agent (RA) respectively. These three agents share many details among each other which results in efficient packet delivery in vehicular communication. Each agent has its own set of responsibilities in building the complete routing protocol. The Figure below depicts the proposed system with three agents. The work of each agent is explained as follows:

\section{Information agent}

The node is elected as information-agent [11] which will in turn have all the neighbor nodes sharing the same information. This information node collects the various information of neighbor nodes and helps to form the cluster. The Information agent has the HELLO message packet which contains the ID, speed and position of every vehicle. This HELLO message packet which is transferred periodically will be broadcasted to all neighbor nodes. After this process, the information agent helps to elect the Cluster agent.

\section{Cluster agent}

The Cluster agent acts as Cluster head, which maintains the cluster topology. Cluster agent is elected based on the neighbor nodes with the information that is provided by the Information agent. Energy consumption is a secondary factor to elect the cluster agent. When the vehicle transmits the messages to longer distance, it needs a higher energy. Whereas, if the cluster agent has low energy then the cluster topology will be destroyed. The energy is calculated based on the distance of the next hop between nodes and neighbor nodes. The final factor is a stability of the cluster topology which is calculated based on the zonal distance between a set of the neighbor nodes. 


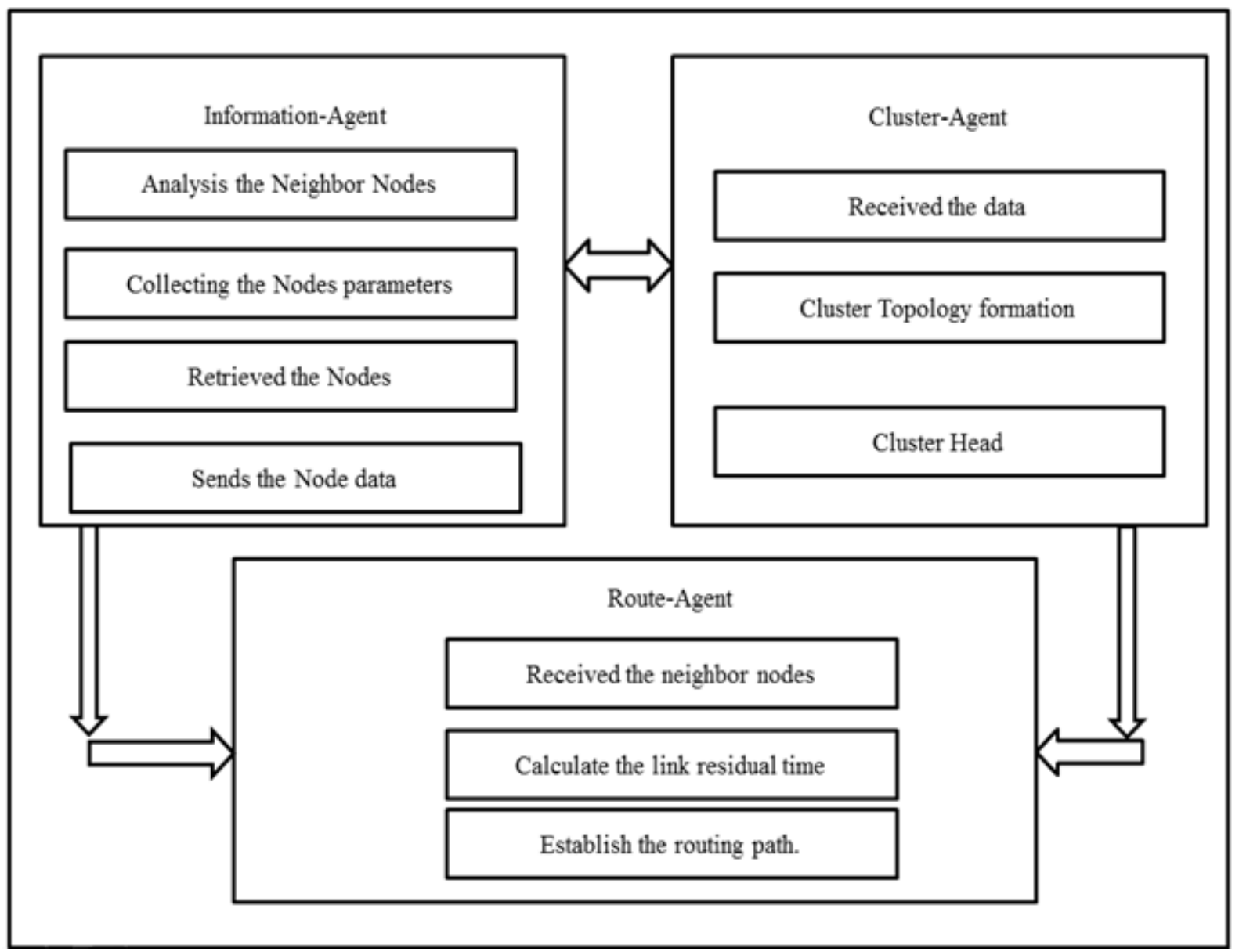

Fig. 1: System Architecture.

\section{Routing agent}

The Routing agent performs the major role in this system; this agent node constructs the routing path for a vehicle communication. Elected Cluster agent and cluster head is notified to the routing agent. In VANET the routing is a one of the major challenge, because of the instability of the nodes and the vehicles are moving with high speed and in various directions. Routing agent is to choose the next hop nodes in the cluster. This next hop node has the maximum link residual time and the low mobility factor compared to other neighbor nodes. The main objective of this routing agent is to calculate the link residual time for all the neighbor nodes [9] and chooses the optimized next hop node for constructing the routing path in vehicular communication. The neighbor node information is provided by information agent.

\section{Algorithm Muti-Agent ()}

Inputs: Vehicle id, speed, direction, position, Signal strength. Output: Information Agent, Cluster Agent,Route Agent and Routing Path construction

\section{Begin}

Information -Agent ()

Begin

Collects the information of neighbor nodes using the HELO message packets.

Retrieve Process

Data communication between IA and CA

End Information Agent.

Cluster-Agent ()

Begin

Calculation ()
Energy consumption (Ec)

Stability Factor (Sf).

Cluster Node weight $(\mathrm{Cw})$.

\}

For each Node

Compare the Cluster Node weights.

End for each.

Declare the CA.

End Cluster Agent.

Route Agent ()

Begin

Data collection from CA and IA.

LRT ()

Construct the routing path.

End Route Agent.

End Algorithm.

The algorithm on the working of the proposed multi-agent routing protocol is given below. The inputs for the algorithm are various parameters of the vehicular network such as vehicle ID, speed, direction, position and signal strength. The output of the algorithm is the final construction of IA, CA, and RA. There are three functions each for IA, CA and RA respectively.

\section{Simulation analysis}

In proposed system, mainly focused on transferring the data from one vehicle to another vehicle with less delay time and high packet delivery ratio. The Adaptive traffic control model was computed using SUMO (Simulation of Urban Mobility) and NS3 (Network Simulator). The SUMO is a simulation tool to create a VANET environment like real time manner. Using this tool, to create a vehicle's movement, in real time scenario. NS3 is used to 
maintain the communication in between vehicles and infrastruc-

ture elements. Table (1) shows the simulation parameters.

Table 1: Simulation Parameters

\begin{tabular}{ll}
\hline Parameters & Value \\
\hline Simulation Time & 360 Seconds \\
Number of Vehicles & 500 \\
Vehicle Communication Range & $1000 \mathrm{~meters}$ \\
Vehicle Speed & $0-100 \mathrm{Km} / \mathrm{hr}$ \\
Data packet Size & $512 \mathrm{bytes}$ \\
MAC Protocol & $802.11 \mathrm{p}$ \\
\hline
\end{tabular}

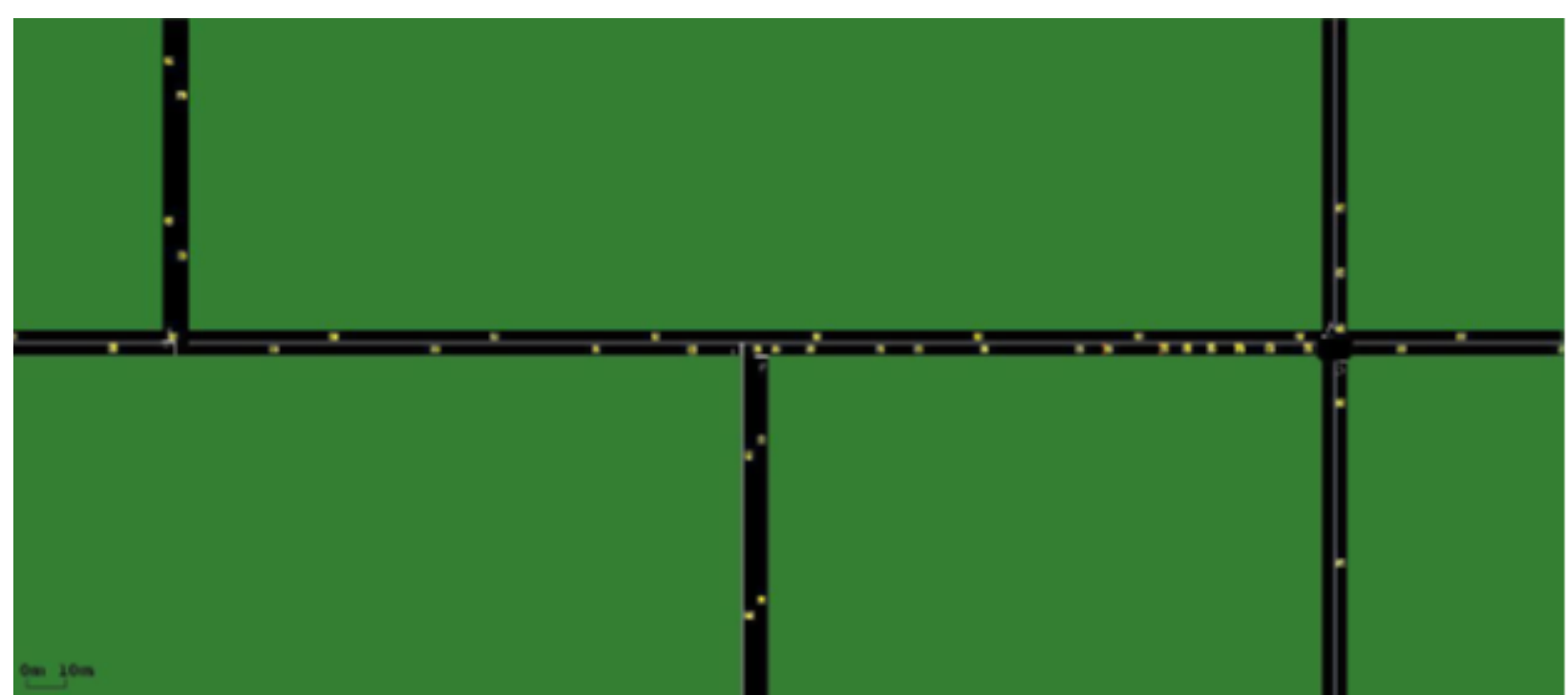

Fig. 2: A) VANET Real Time Environment Setup.

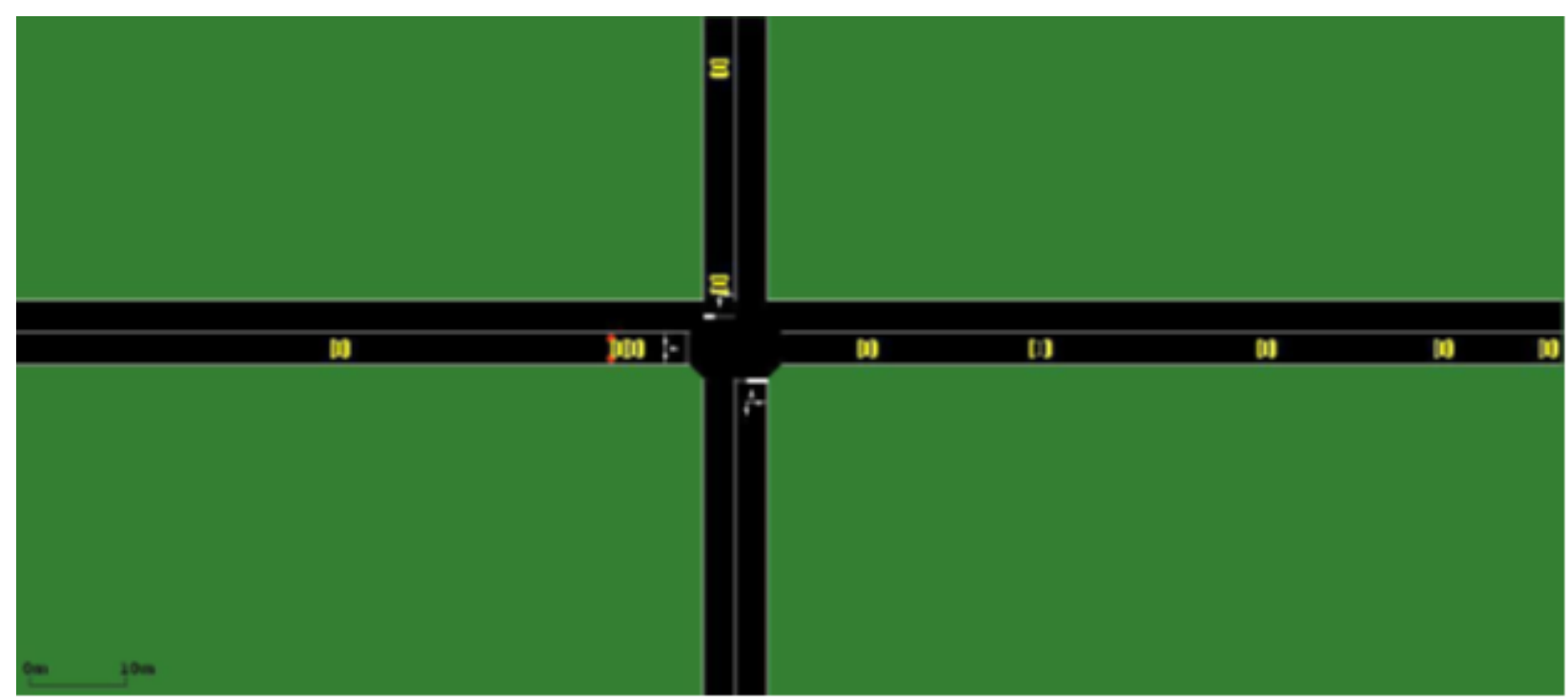

Fig. 2: B) VANET Real Time Environment Setup in Fourway junction.

Fig (2A) and (2B) represents the VANET environment using the SUMO Tool. Its demonstrate the Vehicle movements in a two way lane segment. The vehicles are moving with different speeds and different directions randomly.In this VANET environment the vehicles are moving with different speed and different directions. Fig (3) represents the performance analysis for Packet Delivery Ratio (PDR). Using this ICR multi agent routing method the packet delivery has been increased compared to the Dynamic Source Routing. In ICR multi agent routing method was to reduce the link disconnectivity during the data transmission.
Fig (4) represents the performance analysis for End-to-End delay. Using this ICR multi agent routing method the End-to-End delay has been decreased compared to the Dynamic Source Routing. In ICR multi agent routing method used to transfer the data efficiently with less time period. 


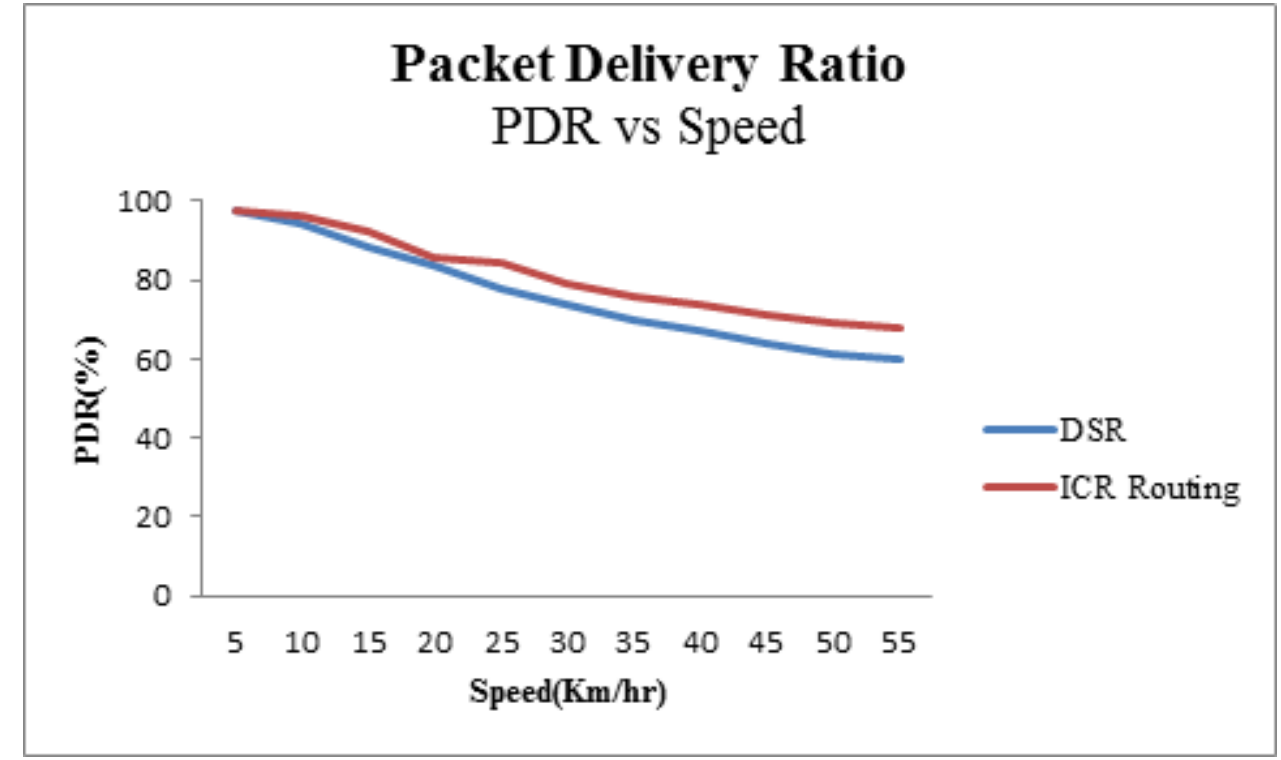

Fig. 3: Packet Delivery Ratio Vs Speed of the Vehicle.

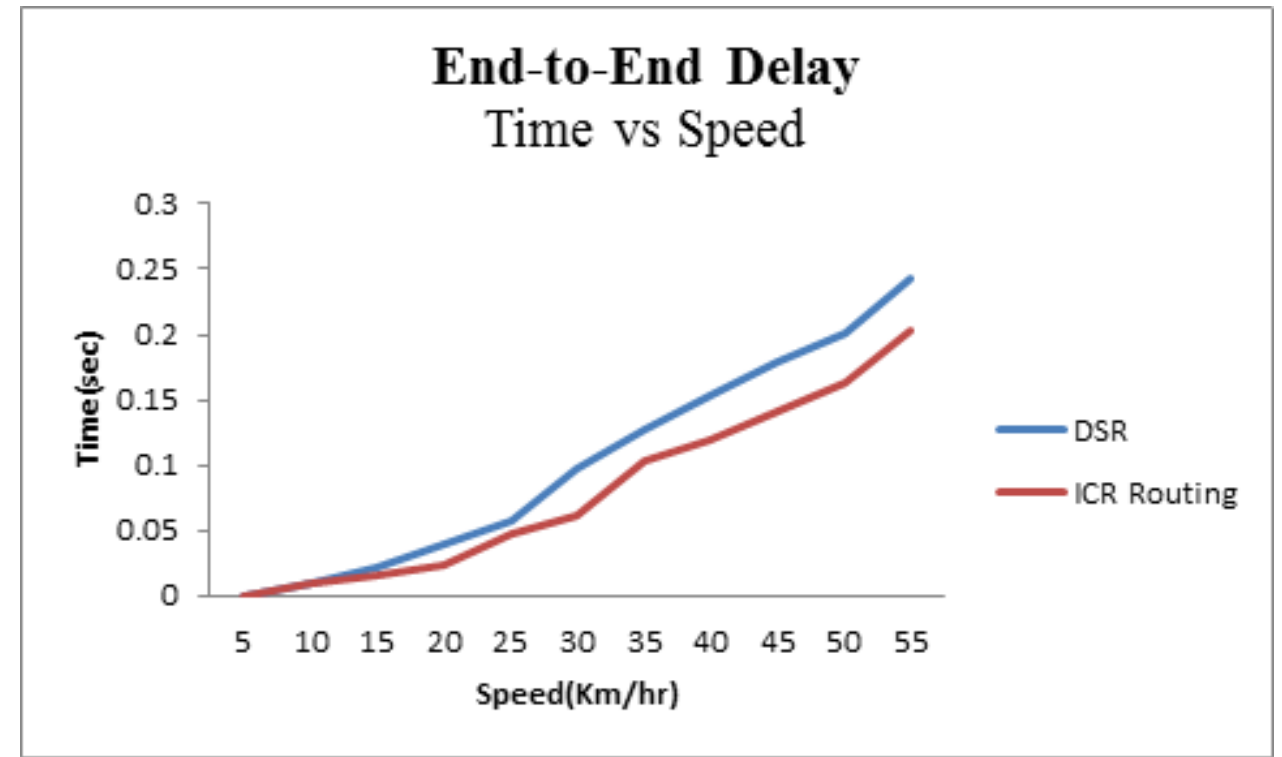

Fig. 4: End to End Delay Vs. Speed.

\section{Conclusion}

No matter how efficient the protocol is, there is always a greater threat of frequent disconnection, unreliable large VANET nodes which are dynamically changing the communication environment. Agent technology takes steps to apply its solution to these networks, which gives a promising solution to the development of routing systems. In this paper, we have successfully proposed a multi agent approach for routing in VANET. This approach has proved to be more efficient than the existing protocols. The IA is to collect the all information; CA maintains the cluster topology and RA is used to construct the efficient routing path. The three agents cooperate together to find the best route possible and also reduces the end-to-end delay and a increased packet delivery ratio.

\section{References}

[1] Weidong Xiang, Richardson, P, Jinhua Guo, "Introduction and Preliminary Experimental Results of Wireless Access for Vehicular Environments (WAVE) Systems", in Proc. 3rd Annual International Conference on Mobile and Ubiquitous Systems - Workshops, 2006, pp. 1-8.

[2] S. Balasubramani and Shankar, "Review on MAC layer delay computation methods in wireless networks," 2017 International Con- ference on Circuit, Power and Computing Technologies (ICCPCT), Kollam, 2017, pp. 1-7

[3] S. Corson, and J. Macker, -Mobile Ad Hoc Networking (MANET): Routing Protocol Performance Issues and Evaluation Considerations, $\|$ RFC editor, 1999.

[4] Balasubramani S, S.K. Rani, K. Suja Rajeswari, "Review on Security Attacks and Mechanism in VANET and MANET", Artificial Intelligence and Evolutionary Computations in Engineering Systems, pp. 655-666, 2016.

[5] Fan Li and Yu Wang, University of North Carolina at Charlotte\|Routing in Vehicular Ad Hoc Networks: A Survey\| IEEE Vehicular magazine,2007,pp-12-22

[6] Balasubramani, Deepalakshmi V. Karthikeyan L, "Comparative Study on Non-Delay Tolerant Routing Protocols in Vehicular Networks", Procedia Computer Science, vol. 50, pp. 252-257, 2015. https://doi.org/10.1016/j.procs.2015.04.052.

[7] BaraaT.Sharef,Raed A. Alsaqour, Mahamod Ismail,\| Vehicular communication Ad-hoc routing protocols: Surveyll, Journal of Networks and Computer Applications, 2014,Vol-40,pp363-396.

[8] Sourav Kumar Bhoi, Pabitra Mohan Khilar," Vehicular Communication Survey" IET Networks, 2014, vol.3, pp.204-217. https://doi.org/10.1049/iet-net.2013.0065.

[9] KarthikeyanL, SelvakumarS, BalasubramaniS" ELRT: Improved Packet Delivery Ratio using Enhanced Link Residual Time in VANET", Advanced in Natural and Applied Science, 2014, pp-2124.

[10] Chatterjee M, Sajal K. Das, and Damla T. WCA: A Weighted Clustering Algorithm for Mobile Ad Hoc Networks. Journal of Cluster 
Computing, Special, Issue on Mobile Ad hoc Networks2002; 5:2; 193-204.

[11] Chen G, Kotz D.A survey of context-aware mobile computing .Research.Technical report, Hanover, NH, USA, 2000.

[12] Mohamed Aissaa, AbdelfettahBelghithb, "Quality of Clustering in mobile Ad Hoc networks",5th International Conference on Ambient Systems, Networks and Technologies (ANT-2014),Procedia Computer Science 32 ( 2014 ) 245 - 252.

[13] Yang Qing , Lim Alvin ,Li Shuang ,Fang Jian And Agrawal Prathima Acar: Adaptive Connectivity Aware Routing For Vehicular Ad Hoc Networks, In City Scenarios Mobile Networks And Applications 2010, 15:1, p. 36-60. https://doi.org/10.1007/s11036-0090169-2.

[14] NeerajBagga., Suraj Sharma, Shubhra Jain and Tushar Ranjan Sahoo, "A Cluster-Tree based Data Dissemination Routing Protocol",

[15] Eleventh International Multi-Conference on Information Processing-2015 (IMCIP-2015),Procedia Computer Science 54 ( 2015 ) $7-13$

[16] H. Wu, R. Fujimoto, R. Guensler, and M. Hunter.MDDV: A Mobility-Centric Data Dissemination Algorithm for Vehicular Networks.
Vehicular
Ad Hoc
Networks,
2004 https://doi.org/10.1145/1023875.1023884.

[17] Neeraj Kumar, Naveen Chilamkurti, Jong Hyuk Park “.Agent learning-based clustering algorithm in vehicular ad hocnetworks". Personal and Ubiquitous Computing, September 2012.

[18] HimanshuTyagi, A. K. Vatsa."Seamless Handoff through Information Retrieval in VANET Using Mobile Agent". IJCSI International Journal of Computer Science July 2011; 8:2; 634-640.

[19] K. Vijayakumar, C.Arun, Automated risk identification using NLP in cloud based development environments, J Ambient Intell Human Computing,DOI 10.1007/s12652-017-0503-7, May 2017.

[20] K.Vijayakumar-C,Arun,Continuous security assessment of cloud based applications using distributed hashing algorithm in SDLC,Cluster Computing DOI 10.1007/s10586-017-1176-x,Sept 2017. 